\title{
p53 expression confers sensitivity to 5-fluorouracil via distinct chromatin accessibility dynamics in human colorectal cancer
}

\author{
CHUL MIN YANG ${ }^{1}$, MOO-KOO KANG ${ }^{2,3}$, WOONG-JAE JUNG ${ }^{2}$, JUNG-SIK JOO ${ }^{2,3}$, \\ YONG-JIN KIM ${ }^{2,3}$, YEEUN CHOI $^{2}$ and HYOUNG-PYO KIM ${ }^{1-3}$ \\ ${ }^{1}$ Severance Biomedical Science Institute; ${ }^{2}$ Department of Environmental Medical Biology, Institute of Tropical Medicine; \\ ${ }^{3}$ Brain Korea 21 PLUS Project for Medical Science, Yonsei University College of Medicine, Seoul 120-752, Republic of Korea
}

Received September 18,2020; Accepted December 3, 2020

DOI: $10.3892 / \mathrm{ol} .2021 .12487$

\begin{abstract}
One of the most commonly used drugs in chemotherapy, 5-fluorouracil (5-FU) has been shown to be effective in only $10-15 \%$ of patients with colon cancer. Thus, studies of the mechanisms affecting 5-FU sensitivity in these patients are necessary. The tumor suppressor protein p53 is a transcription factor that serves important roles in cell apoptosis by regulating the cell cycle. It has also been characterized as a key factor influencing drug sensitivity. Furthermore, accessible chromatin is a hallmark of active DNA regulatory elements and functions as a crucial epigenetic factor regulating cancer mechanisms. The present study assessed the genetic regulatory landscape in colon cancer by performing RNA sequencing and Assay for Transposase-Accessible Chromatin sequencing, and investigated the effects of 5-FU on chromatin accessibility and gene expression. Notably, while treatment with 5-FU mediated global increases in chromatin accessibility, chromatin organization in several genomic regions differed depending on the expression status of p53. Since the occupancy of p53 does not overlap with accessible chromatin regions, the 5-FU-mediated changes in chromatin accessibility were not regulated by direct binding of p53. In the p53-expressing condition, the 5-FU-mediated accessible chromatin region was primarily associated with genes encoding cell death pathways. Additionally, 5-FU was revealed to induce open chromatin conformation at regions containing binding motifs for AP-1 family transcription factors, which may drive expression of apoptosis pathway genes. In conclusion, expression of p53 may confer 5-FU sensitivity by regulating chromatin accessibility of distinct genes associated with cell apoptosis in a transcription-independent manner.
\end{abstract}

Correspondence to: Dr Hyoung-Pyo Kim, Department of Environmental Medical Biology, Institute of Tropical Medicine, Yonsei University College of Medicine, 50-1 Yonsei-ro, Seodaemun, Seoul 120-752, Republic of Korea

E-mail:kimhp@yuhs.ac

Key words: 5-fluorouracil, chromatin accessibility, drug sensitivity, p53, apoptosis

\section{Introduction}

Colorectal cancer ranks as the second most deadly cancer among all cancer types, and colorectal cancers are commonly treated with 5-fluorouracil (5-FU), anticancer drug. 5-FU is known to interfere with DNA synthesis in cancer cells by inhibiting thymidylate synthase, as a mechanism that inhibits cell proliferation and leads to cell death (1-3). Although 5-FU plays a central role in the treatment of colorectal cancer, it elicits any effect in only 10-15\% of colorectal cancer patients, and approximately 40-50\% of treatments are successful upon combination therapy with other antitumor drugs, including oxaliplatin, leucovorin, and others. Therefore, outlining the mechanisms underlying sensitivity to 5-FU among colorectal cancer cells would be helpful.

Cancer research has shown p53 to be a tumor suppressor, and 553 dysfunction has been found to lead to the development of colorectal cancer. Moreover, p53 has been characterized a key factor influencing drug sensitivity: p53 overexpression has been shown to increase the effect of chemotherapy in patients with stage III colorectal cancer with mutant p53 (4-6). The major biological function of p53 is to initiate cell apoptosis by regulating the cell cycle at the G1/S and the G2/M check points. Interestingly, p53 studies have indicated that active p53 exhibits both transcriptional and non-transcriptional activity and that both can affect apoptosis. Under various cell death conditions, the non-transcriptional activity of p53 induces mitochondrial outer membrane permeabilization (MOMP), and this triggers the release of pro-apoptotic factors from the mitochondrial inter-membrane space (7-9).

Chromatin structure and organization influence the identity of cells by regulating cell-specific signaling networks $(10,11)$, and recently, various epigenetic studies have reported a link between alterations in chromatin structure and cancer progression (12-15). Measurement of chromatin accessibility during prostate cancer progression revealed that chromatin relaxation increases the binding of oncogenic transcription factors, resulting in increased expression of tumor growth-related genes $(16,17)$. Another report showed that TGF- $\beta$ induces chromatin accessibility to cis-regulatory elements of epithelial-to-mesenchymal transition-related genes, including $C D H 1, C D H 2$, and VIM. Additionally, research has shown that TGF- $\beta$ induced chromatin accessibility increases the 
transcriptional activity of RUNX, AP-1, and Etv4/5, and results in epithelial-to-mesenchymal transition in mammary gland epithelial cells (18). Altogether, these studies indicate that external stimuli can affect chromatin accessibility and that such effects are strongly related with stimulus-mediated cellular phenotypes.

While it is known that p53 plays a key role in successful chemotherapy, the mechanisms of how p53 affects 5-FU sensitivity remain unknown. Rubbi and Milner (19) showed that ultraviolet radiation induces p53-mediated global chromatin relaxation. However, the authors did not demonstrate the effects of global chromatin relaxation on DNA damage-induced cell death (19). Therefore, in this study, we hypothesized that since 5-FU is also a DNA-damaging reagent, like ultraviolet radiation, TP53-wild-type (TP53-WT) and TP53-knockout (TP53-KO) colorectal cancer cells would show differences in gene expression at chromatin accessible regions under 5-FU treatment and that such differences would affect 5-FU sensitivity depending on p53 status. To prove our hypothesis, we performed Assay for Transposase-Accessible Chromatin sequencing (ATAC-seq) to assess chromatin accessibility and RNA sequencing (RNA-seq) to measure associated gene expression in both TP53-WT and TP53-KO colorectal cancer cells exposed to 5-FU treatment.

\section{Materials and methods}

Cell culture. The human colorectal carcinoma cell line HCT116 TP53-WT and HCT116 TP53-KO, initially described by Bunz et al (20), were obtained as a kind gift from Dr Jae-Hoon Jeong (Korea Institute of Radiological and Medical Sciences, Korea). The genetic background of TP53-WT and TP53-KO were verified by performing genotyping using PCR primers (Table SI), which amplify a 220-bp band for TP53 exon 2 and a 519-bp band for CTCF exon 12 as the control (Fig. S1). Target DNA for genotyping was amplified using Dr. Taq DNA Polymerase (Allforlab) with initial denaturation at $95^{\circ} \mathrm{C}$ for $3 \mathrm{~min}$, followed by 35 cycles of denaturation at $95^{\circ} \mathrm{C}$ for $10 \mathrm{sec}$, annealing at $63^{\circ} \mathrm{C}$ for $30 \mathrm{sec}$, extension at $72^{\circ} \mathrm{C}$ for $1 \mathrm{~min}$, and a final elongation step at $72^{\circ} \mathrm{C}$ for $5 \mathrm{~min}$. Cells were cultured in Roswell Park Memorial Institute 1640 medium (Hyclone) supplemented with $10 \%$ fetal bovine serum (Hyclone) and $1 \%$ penicillin/ streptomycin (100 U/ml:100 $\mu \mathrm{g} / \mathrm{ml}$; Hyclone) and were grown at $37^{\circ} \mathrm{C}, 5 \% \mathrm{CO}_{2}$, and $98 \%$ humidity.

Cell viability assay. Cell viability was analyzed using CellTiter Glo (Promega, G7570) according to the manufacturer's instructions. Luminescence was measured using a microplate luminometer (Berthold Centro XS3 LB 960) and MikroWin2000 software.

ChIPmentation library preparation. Chromatin immunoprecipitation followed by sequencing (ChIP-seq) assays were performed using p53 antibodies (Santa Cruz Biotechnology, Inc.; cat. no. sc-126) as described previously (21) with minor modifications. ChIP-seq libraries were prepared using a Nextera DNA Sample Prep kit (Illumina, Inc.) according to the manufacturer's instructions. Libraries were sequenced on an Illumina HiSeq 2500 system, generating 100 bp paired-end reads.
RNA-seq library preparation. Total RNA was isolated using Hybrid-R Total RNA kits (GeneAll Biotechnology Co. Ltd.), and rRNAs were depleted by using the NEBNext rRNA Depletion kit (NEB; cat. no. E6350). RNA sequencing (RNA-seq) libraries was prepared using NEBNext Ultra RNA library prep kits (NEB; cat. no. 7770) according to the manufacturer's instructions. Libraries were sequenced on an Illumina HiSeq 2500 system, generating 100 bp paired-end reads.

ATAC-seq library preparation. Assay for TransposaseAccessible Chromatin sequencing (ATAC-seq) libraries were prepared as described previously (22) and sequenced on an Illumina HiSeq 2500 system, generating 100 bp paired-end reads.

ChIP-seq data processing. Raw reads were trimmed using trim galore with default parameters to remove low quality and adapter sequences. Trimmed reads were mapped to the human genome reference hg19 using BWA (23). The reference genome was downloaded from GENCODE V19. Reads with low mapping quality were filtered using SAMtools (24). MAPQ $<30$ were used to obtain uniquely mapped reads. Duplicated reads were filtered using Picard (Picard version 2.23.9; https://broadinstitute.github.io/picard). Reads mapped to mitochondrial chromosomes were filtered. Peaks were called using MACS2 (25) with input DNA as a control.

ATAC-seq data processing. Raw reads were trimmed using trim galore with the same default parameters as in ChIP-seq analysis. Trimmed reads were mapped to the hg19 reference genome using BWA (23). Fragments below 2,000 bp were allowed. Reads with low mapping quality were filtered using SAMtools (24). Duplicated reads and mitochondrial chromosomes were filtered as described above. Since Tn5 transposase binds as a dimer and inserts two adaptors separated by $9 \mathrm{bp}$, all aligned reads were shifted by +4 bp on the positive strand and -5 bp on the negative strand using the deepTools (26) function alignmentSieve. To obtain nucleosome free regions, fragments over $140 \mathrm{bp}$ in length were filtered out. ATAC-seq peak calling was performed using MACS2 (25). ATAC-seq peaks across samples were merged using mergePeaks, and differential peaks were called using Homer getDifferentialPeaks.pl (Homer version 4.11; http://homer.ucsd.edu). Overlapping of open chromatin regions with enrichment of p53 was analyzed using bedtools (27) intersect: Overlap of at least 1-bp intervals was counted. Homer annotatePeaks.pl was used to annotate differential ATAC-seq peaks to their nearest genes. Homer findMotifsGenome.pl. was used to identify motifs enriched at open chromatin regions. ATAC-seq and ChIP-seq data were visualized using the Integrative Genomics Viewer (28).

RNA-seq data processing. Raw reads were trimmed using trim galore. For transcript abundance quantification analysis, STAR (29) was used to map the reads to the human genome. RSEM (30) was used to quantify transcripts. Differentially expressed genes between groups were calculated using DESeq2 $\mathrm{R}$ package (31). Genes with low read counts were filtered out before differential analysis. DESeq2 provides a statistical analysis method and assessment of differential expression using a model based on negative binomial distribution. Gene 
ontology and pathway enrichment analyses were performed using the Enrichr online tool $(32,33)$.

Statistics. All experimental values are presented as mean \pm SD. Cell viability assays were performed in triplicate, and RNA-seq and ATAC-seq experiments were performed in duplicate. Most of statistical significance was calculated by two-way analysis of variance, in which $\mathrm{P}<0.05$ was considered statistically significant (GraphPad Prism 7 software; GraphPad Software, Inc.). Post hoc multiple comparisons were followed by Bonferroni correction. Two groups were compared using unpaired Student's t-test, in which $\mathrm{P}<0.001$ was considered statistically significant ( $\mathrm{R}$ version 4.0.3; The R Foundation).

\section{Results}

Chromatin accessibility changes in response to 5-FU depending on p53 status. Responding to drug stimulation, p53 has been shown to increase drug sensitivity in various cancer cells $(34,35)$. In order to investigate the effects of p53 on responses to 5-FU among colorectal cancer cells (HCT116 cells), we first treated TP53-WT and TP53-KO HCT116 cells with 5-FU and then measured mRNA expression levels of p53 using RNA-seq analysis. As expected, we noted that p53 expression increased under 5-FU treatment at $24 \mathrm{~h}$ in TP53-WT cells, but not TP53-KO cells (Fig. 1A). Next, we performed cell viability assay and found that the presence of p53 conferred sensitivity to 5-FU among HCT116 cells (Fig. 1B), clearly confirming the role of p53 in 5-FU sensitivity in HCT116 cells.

Physical DNA accessibility is important in establishing and maintaining cellular identity through the regulation of the expression of various genes $(36,37)$. Moreover, recent research has shown that drug-mediated cellular replication stress induces chromatin remodeling and that such modification is highly associated with drug responses in cells $(38,39)$. To ascertain the effects of 5-FU on chromatin accessibility, we performed ATAC-seq before and after 5-FU treatment, and found that global chromatin accessibility increased around 3 -fold under 5-FU treatment for $24 \mathrm{~h}$ in both TP53-WT and TP53-KO cells (Fig. 1C). Next, we analyzed regions showing differences in chromatin accessibility between TP53-WT and TP53-KO cells after 5-FU treatment to evaluate the role of p53 in 5-FU-mediated chromatin remodeling. When we merged and compared all ATAC-seq peaks called from 5-FU-treated TP53-WT and TP53-KO cells, we found that chromatin accessibility was equal at 5152 regions in the cells under 5-FU treatment regardless of the presence of TP53 (Fig. 1D). Respectively, however, 5-FU elicited higher chromatin accessibility at 9,131 regions in TP53-WT cells and at 9,900 regions in TP-53-KO cells (Fig. 1D). These data indicated that the expression status of p53 influences chromatin accessibility in response to 5-FU.

It is well known that p53 functions as a transcription factor that binds to specific DNA sequences to trans-activate a number of genes $(40,41)$. To determine whether p53 impacts 5 -FU-mediated changes in chromatin accessibility by directly binding to regulatory regions of DNA, we performed chromatin immunoprecipitation followed by sequencing (ChIP-seq) to analyze the distribution of binding sites for p53 in TP53-WT cells. Consistent with a previous report (42), most TP53 binding regions were located in inaccessible chromatin regions, and only $1.4 \%$ of 5-FU-induced accessible regions overlapped with TP53 binding sites (Fig. 1E). These results demonstrated that p53 influences 5-FU-mediated changes in chromatin accessibility in a transcription-independent manner.

Changes in chromatin accessibility are associated with distinct gene expression profiles in response to 5-FU. Next, we explored genome-wide changes in gene expression profiles following 5-FU treatment by performing RNA-seq. In response to 5-FU treatment, a larger number of differentially expressed genes (DEGs, fold change $>2$, P-value $<0.05$ ) in TP53-WT cells than in TP53-KO cells (1693 genes in TP53-WT versus 583 genes in TP53-KO) (Fig. 2A). Interestingly, 772 and 281 DEGs from TP53-WT and TP53-KO cells, respectively, were directly associated with 5-FU-induced changes in chromatin accessibility (Fig. 2A). Moreover, expression of 551 and 150 genes were significantly increased following 5 -FU treatment in TP53-WT and TP53-KO cells, respectively (Fig. 2B). Gene ontology analysis for 5-FU-mediated upregulated genes harboring differentially accessible regions (DARs) in TP53-WT cells revealed significant enrichment of genes known to be associated with p53 pathways, apoptosis, and genotoxicity (Fig. 2C), while those in TP53-KO cells showed enrichment of genes associated with cancer progression, including senescence and autophagy, circadian rhythm-related genes, melatonin metabolism, and PI3K-AKT-mTOR signaling (Fig. 2D). Moreover, we found that 135 genes were overlapped among the genes whose expressions and chromatin accessibilities were changed after 5-FU treatment in both TP53-WT and TP53-KO cells. Gene ontology analysis of these overlapped genes revealed significant enrichment of genes associated with transcription regulation, cell differentiation regulation and apoptosis (Fig. S2). These results indicated that changes in chromatin accessibility in response to 5-FU elicit distinct gene expression profiles in colorectal cancer cells depending on p53 status.

Specific chromatin accessible regions in TP53-WT cells are highly associated with expression of cell death-related genes. Among the regions showing higher 5-FU-mediated chromatin accessibility in TP53-WT cells, compared to TP53-KO cells (Figs. 1D and 3A), we selected DARs that exhibited a greater than two-fold increase in TP53-WT cells, with an adjusted P-value of less than 0.05 . The annotation of these TP53-WT-specific DARs to their nearest genes allowed us to identify 95 genes with significantly higher expression in TP53-WT cells than TP53-KO cells (Fig. 3B; Table SII). Gene ontology analysis demonstrated that these TP53-WT-specific upregulated genes were significantly enriched in cell death pathways (Table I). As a representative example, Fig. 3C shows that chromatin accessibility in the promoter regions of two tumor suppressor genes, SEMA3B and PHLDA3, as well as their mRNA levels, significantly increased in response to 5-FU treatment in TP53-WT cells only. De novo transcription factor binding motif analysis revealed that AP-1 complex motifs were enriched at the TP53-WT specific chromatin accessible regions $\left(\mathrm{P}=1 \times 10^{-31}\right)$ (Fig. 3D). AP-1 complex is a well 
A

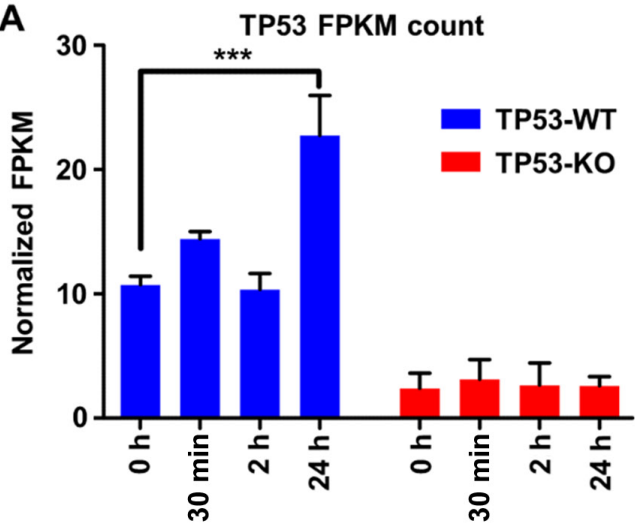

5-FU treatment time point

C

Chromatin accessible regions

TP53-WT

TP53-KO

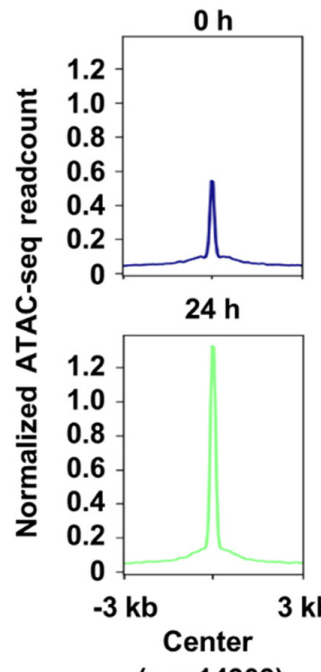

$(n=14303)$
$\mathrm{Oh}$

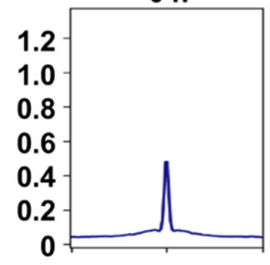

$24 \mathrm{~h}$

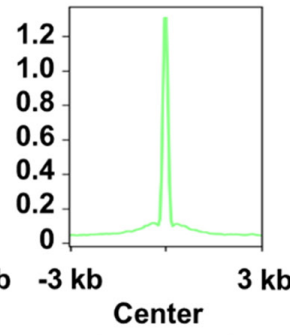

$(n=15092)$

E

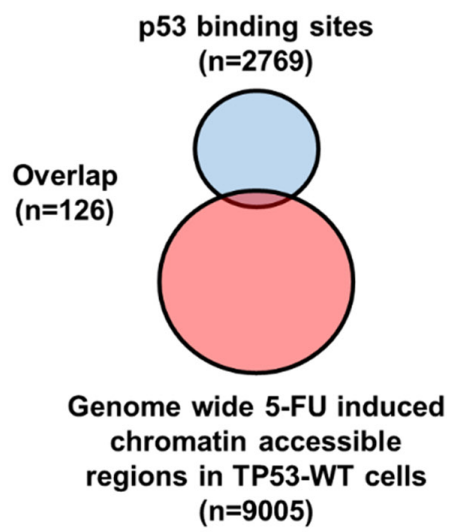

B

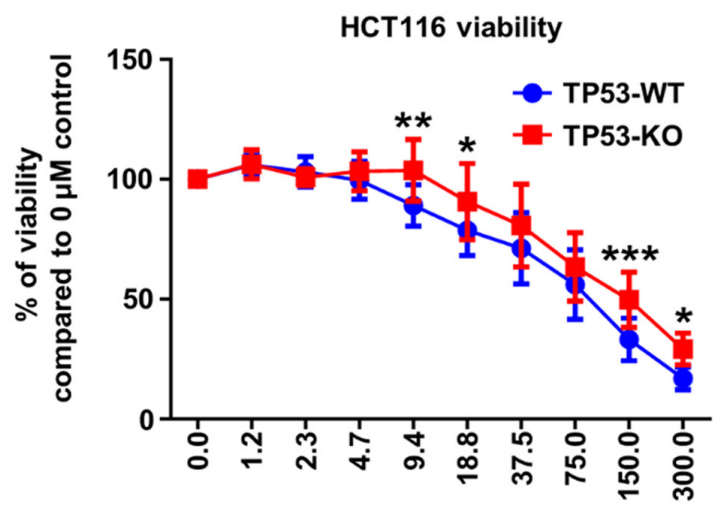

5-FU concentration $(\mu \mathrm{M})$

D Heatmap intensity

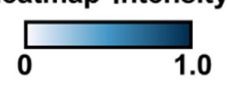

5 -FU induced common DARs

( $n=5152)$

5-FU induced TP53-WT enriched DARs ( $n=9131)$

5-FU induced TP53-KO enriched DARs ( $n=9900$ )

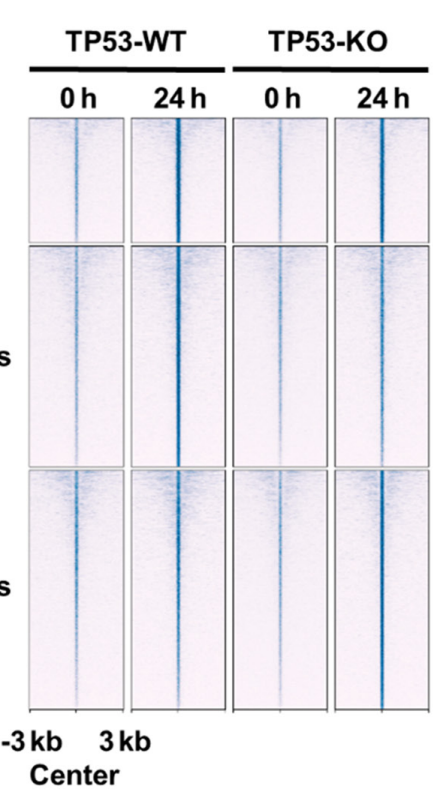

5-FU induced DARs p53 dependent
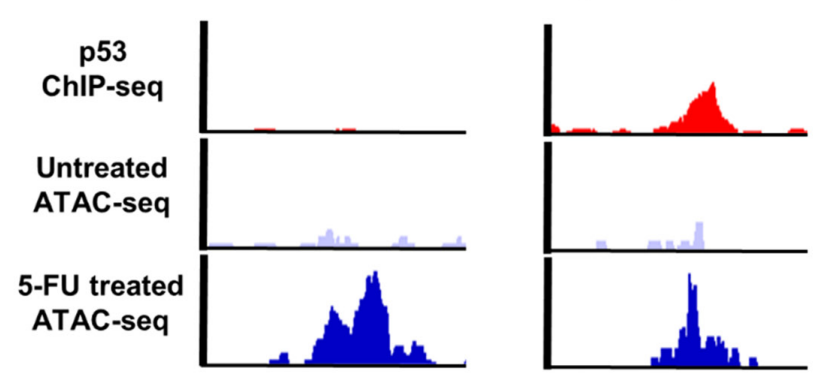

Figure 1. Distinct changes in chromatin accessibility in response to 5-FU depending on p53 status (A) TP53 mRNA expression levels were measured by RNA-seq for TP53-WT or -KO cells treated with $300 \mu \mathrm{M} 5$-FU for the indicated time points (n=2 per group). (B) The viability of TP53-WT and TP53-KO cells measured $72 \mathrm{~h}$ after treatment of various concentration of 5-FU ( $\mathrm{n}=6$ per group). (C) Histogram showing the average tag density of ATAC-seq peaks called for TP53-WT and TP53-KO cells before and after treatment with 5-FU for $24 \mathrm{~h}$. (D) Heatmap showing differentially accessible chromatin regions in TP53-WT and TP53-KO cells before and after treatment with 5-FU for $24 \mathrm{~h}$. (E) Venn diagram showing the overlap of TP53 ChIP-seq peaks and ATAC-seq peaks in TP53-WT cells treated with 5-FU for $24 \mathrm{~h}$ (left), and a genomic snapshot depicting representative DARs (right). The data are presented as a mean \pm SD. ${ }^{*} \mathrm{P}<0.05 ;{ }^{* *} \mathrm{P}<0.01 ;{ }^{* * *} \mathrm{P}<0.001$. DARs, differentially accessible regions; TP53-WT, TP53-wild-type; TP53-KO, TP53-knockout.

characterized transcription factor family known to be activated in response to various cellular stress signals, and research has shown that it regulates various genes related with proliferation, immune response, and cell death (43). One of the AP-1 family transcription factors in particular, c-Jun, has been found to inhibit cell proliferation and to induce apoptosis in response to genotoxic stress by increasing caspase activity (44-46). These results suggest that, unlike in TP53-KO cells, 5-FU elicits 
A

5-FU induced DEGs

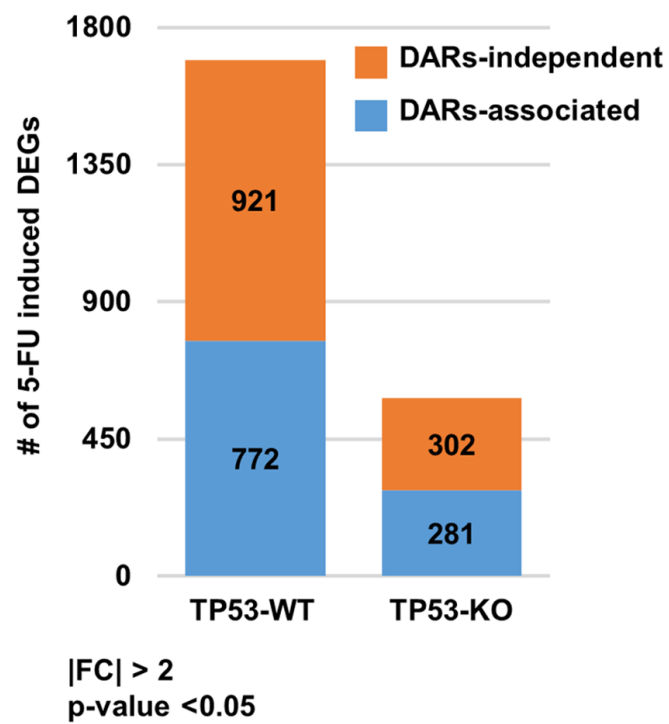

B

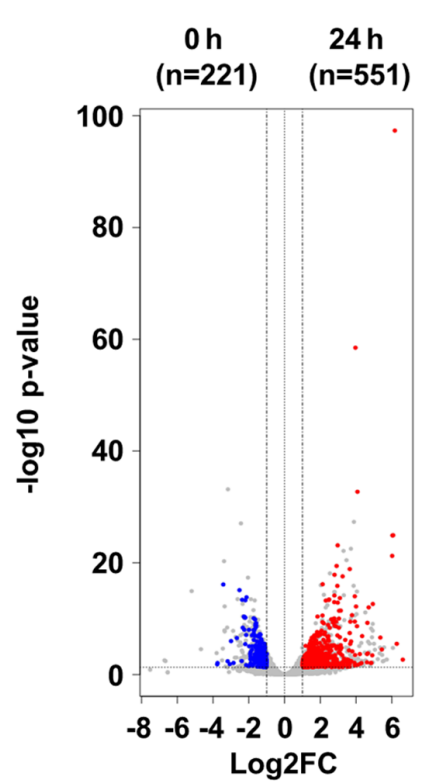

TP53-KO

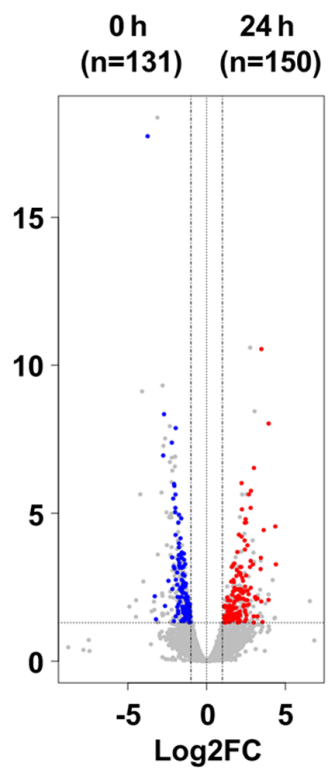

C

Upregulated in TP53-WT $(n=551)$

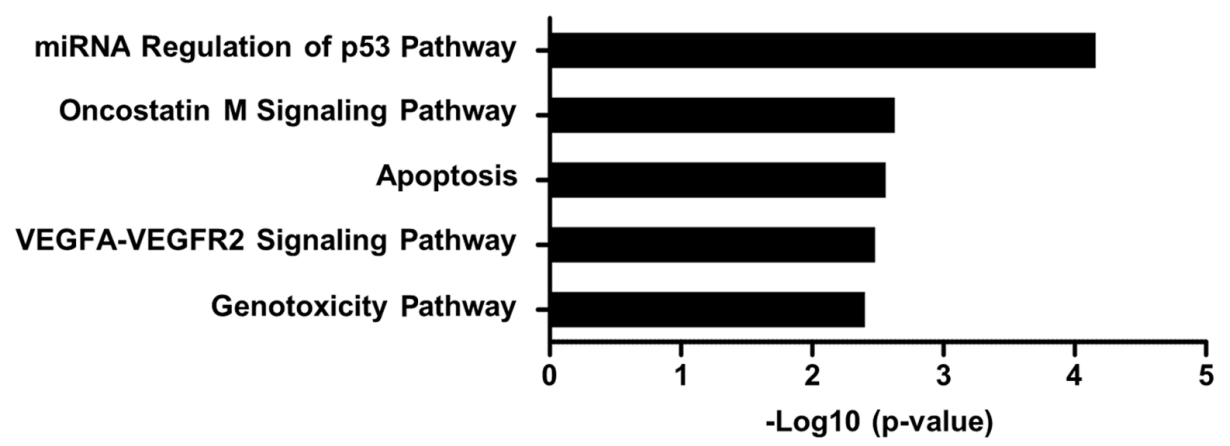

D

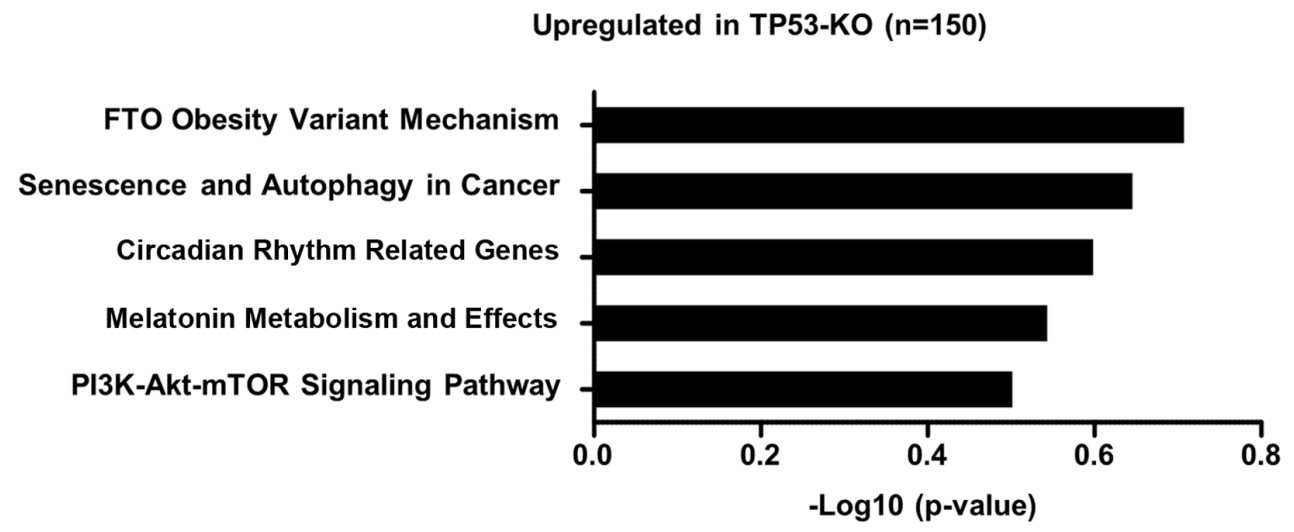

Figure 2. Differential changes in chromatin accessibility depending on p53 status are associate with distinct gene expression profiles in response to 5-FU. (A) Differentially expressed genes (DEGs) that exhibited greater than two-fold changes with an adjusted P-value of less than 0.05 are denoted in TP53-WT and TP53-KO cells based on the association with 5-FU-induced chromatin accessibility. (B) Volcano plot showing DEGs associated with 5-FU-induced DARs in TP53-WT (left) and TP53-KO (right) cells. (C) and (D) Pathway enrichment analysis for the genes that showed both higher mRNA expression and greater chromatin accessibility upon 5-FU treatment for $24 \mathrm{~h}$ in TP53-WT cells (C) or TP53-KO cells (D). TP53-WT, TP53-wild-type; TP53-KO, TP53-knockout.

increases in chromatin accessibility at specific regions in TP53-WT cells that promote the binding of AP-1 transcription factor to genes related to cell death, which may contribute to the higher sensitivity of TP53-WT cells to 5-FU.

\section{Discussion}

Modification of the structure of chromatin can affect the expression of various genes, and it results in changing 
A

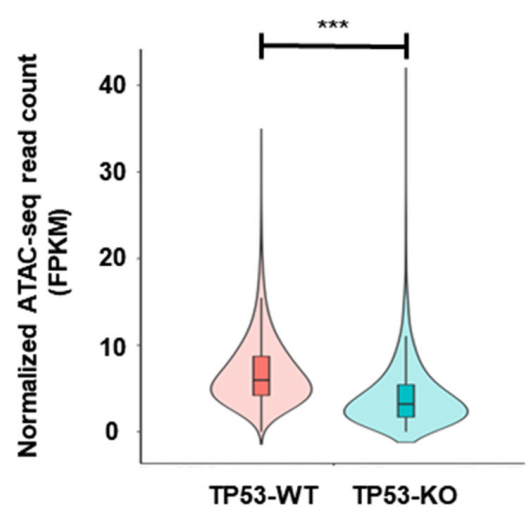

C
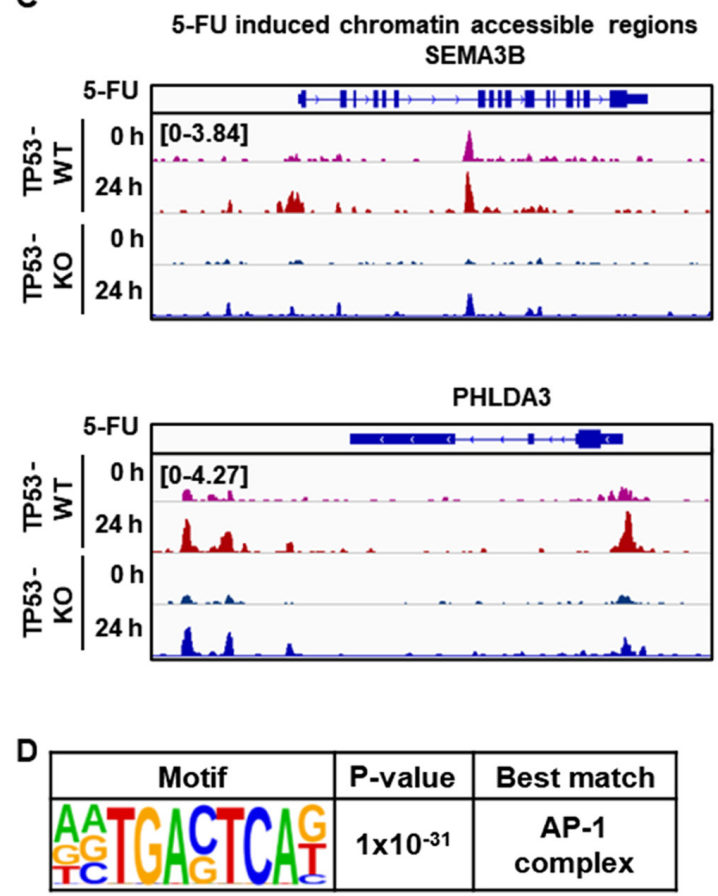

B
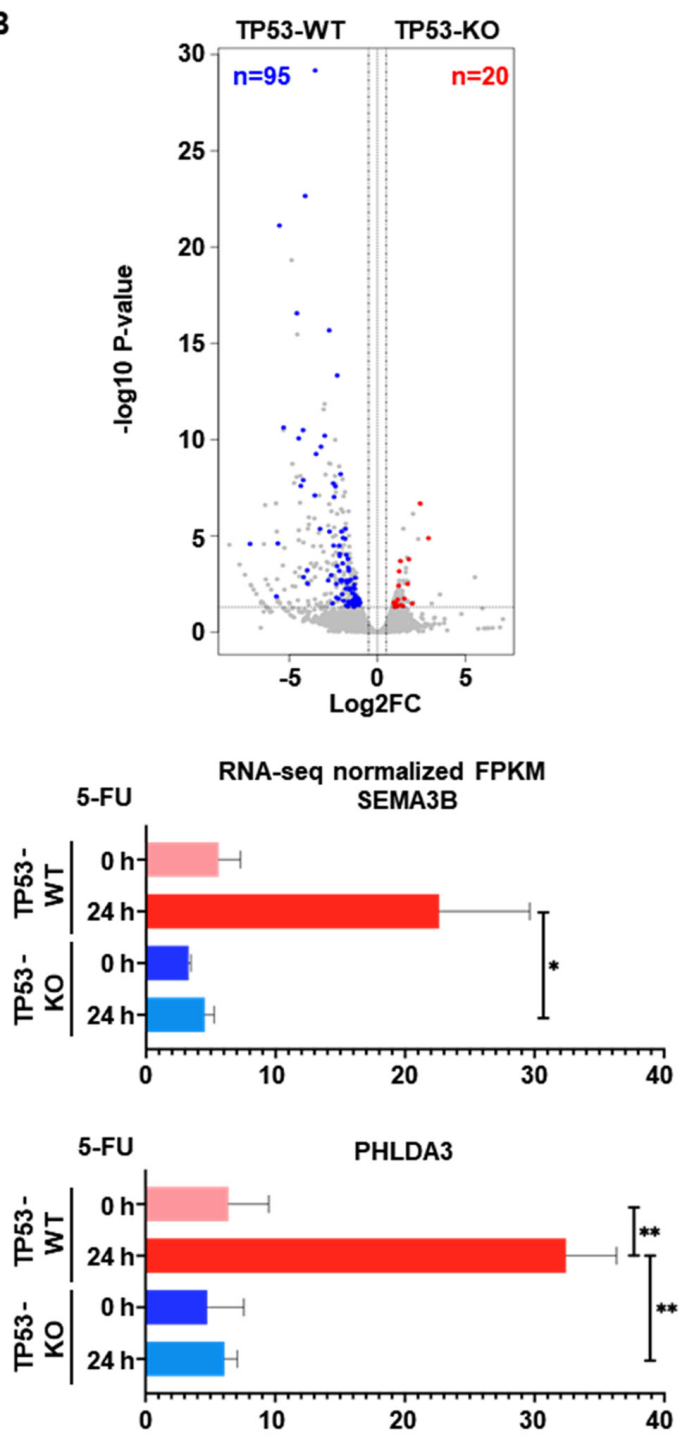

Figure 3. Distinct 5-FU-induced gene expression is associated with higher chromatin accessibility in TP53-WT cells than TP53-KO cells. (A) Violin plot showing 9,131 DARs with higher 5-FU-induced chromatin accessibility in TP53-WT cells, compared to TP53-KO cells ( $\mathrm{n}=2$ per group, $\left.{ }^{* * * *} \mathrm{P}<0.001\right)$. (B) Volcano plot showing DEGs associated with 5-FU-induced DARs that exhibited a greater than two-fold increase in TP53-WT cells, with an adjusted P-value of less than 0.05. The number of genes that exhibited greater than two-fold increases in TP53-WT (blue) and TP53-KO (red) cells with a P-value of less than 0.05 are indicated. (C) Genomic snapshot of the SEMA3B (upper left) and PHLDA3 (lower left) genes showing the densities of ATAC-seq reads in TP53-WT and TP53-KO cells before and after 5-FU treatment for $24 \mathrm{~h}$. Bar graph shows the mRNA expression levels of SEMA3B (upper right) and PHLDA3 (lower right) genes measured by RNA-seq from TP53-WT and TP53-KO cells before and after 5-FU treatment for $24 \mathrm{~h}(\mathrm{n}=2$ per group, "P<0.05; "* $\mathrm{P}<0.01)$. (D) Enriched de novo motif analysis for the DARs that showed both higher mRNA expression and greater chromatin accessibility in TP53-WT cells, compared to TP53-KO cells, after 5-FU treatment for $24 \mathrm{~h}$. DARs, differentially accessible regions; TP53-WT, TP53-wild-type; TP53-KO, TP53-knockout.

molecular signaling networks influencing cellular phenotypes. According to the research about the chromatin accessibility and architecture, extracellular stimulation remodels chromatin structure, and it results in modification of cellular signaling networks toward unique cellular phenotypes $(18,47)$. In this study, we found through RNA- and ATAC-seq that 5-FU increases chromatin accessibility in colorectal cancer cells (HCT116 cells) and that the regions of increased chromatin accessibility induced by 5 -FU differed depending on p53 status: In cells expressing p53, 5-FU elicited increased chromatin accessibility in genes important to p53 pathways, apoptosis, and genotoxicity, whereas in cells not expressing p53, 5-FU elicited increased chromatin accessibility in genes important to cancer progression.

ATAC-seq is one of the best experimental techniques with which to measure chromatin accessibility, and it allows researchers to predict main transcription factors in cis-regulatory regions. In this study, we used ATAC-seq to prove our hypothesis that the sensitivity among colorectal cancer cells to 5-FU may be due to chromatin accessibility and p53 status. 
Table I. GO analysis for the 95 TP53-WT-specific upregulated genes (related with Fig. 3B).

\begin{tabular}{lll}
\hline GO biological process & Fold enrichment & P-value \\
\hline Metal ion transport (GO:0030001) & 4.84 & $1.17 \times 10^{-2}$ \\
Regulation of cell adhesion (GO:0030155) & 4.33 & $4.25 \times 10^{-2}$ \\
Regulation of cell death (GO:0010941) & 2.99 & $1.51 \times 10^{-2}$ \\
Cellular response to chemical stimulus (GO:0070887) & 2.25 & $9.35 \times 10^{-4}$ \\
Regulation of cell communication (GO:0010646) & 2.23 & $6.93 \times 10^{-3}$ \\
Regulation of signaling (GO:0023051) & 2.16 & $7.96 \times 10^{-3}$ \\
Response to chemical (GO:0042221) & 2 & $8.63 \times 10^{-4}$ \\
Negative regulation of cellular process (GO:0048523) & 1.9 & $8.91 \times 10^{-3}$ \\
Cell communication (GO:0007154) & 1.88 \\
Negative regulation of biological process (GO:0048519) & 1.86 \\
Cellular response to stimulus (GO:0051716) & 1.85 & $8.53 \times 10^{-3}$ \\
Signaling (GO:0023052) & 1.76 & $1.49 \times 10^{-2}$ \\
Response to stimulus (GO:0050896) & 1.4 & $2.72 \times 10^{-4}$ \\
Biological regulation (GO:0065007) & $2.90 \times 10^{-2}$ \\
\end{tabular}

ATAC-seq clearly showed that 5-FU increased chromatin accessibility in HCT116 cells (Fig. 1C and D). Noteworthy, 5-FU induced chromatin accessibility differed depending on p53 status in HCT116 cells (Fig. 1D). To identify p53 activities related with 5-FU induced chromatin accessibility, we compared p53 ChIP-seq and ATAC-seq results under 5-FU treatment in TP53-WT cells. From the integration analysis, we found that the difference in chromatin accessibility was not the direct effect of p53 binding: $96 \%$ of p53 binding regions were located in chromatin inaccessible regions (Fig. 1E). Thus, we deemed that the effects of p53 on 5-FU-induced chromatin accessibility in TP53-WT cells are related with non-transcriptional activities. Studies of p53 in relation to drug sensitivity have primarily focused on correlations between signaling networks and p53 status, such as genetic mutation and gene silencing, and most have focused solely on the transcriptional activities of p53 $(34,48,49)$. Nonetheless, a few studies have recently described the effects of p53 non-transcriptional activity on apoptosis. Although these studies only reported on MOMP (7-9), their results, together with ours, indicate that p53 may carry out several non-transcriptional functions that await discovery. Given the high incidence of TP53 mutation in many different types of cancer, it would be interesting to explore the role of p53 in drug sensitivity and chromatin structure in diverse cancer cells, which is beyond the scope of the current study but remains to be our next project to pursue.

In motif analysis of regions showing increased chromatin accessibility upon treatment with 5-FU in TP53-WT cells, we detected the AP-1 complex binding motif (Fig. 3D). Various contradictory functions have been reported for AP-1 complex: It generally induces cell proliferation (50) and differentiation (51); however, one study has indicated that it also shows an apoptotic function (45). Of note, the contradictory functions of AP-1, especially c-JUN, appear to depend on the cellular context and extracellular stimuli (52). Although the function of AP-1 complex in relation to apoptotic signaling is still controversial and unclear, we presume that, through non-transcriptional activity, p53 increases the accessibility of AP-1 binding regions in response to treatment with 5-FU in TP53-WT cells, resulting in activation of apoptotic signaling pathways, thereby conferring 5-FU sensitivity. Unfortunately, however, since motif analysis is only useful in predicting possible binding proteins based on genomic sequences, we are unable to confirm the direct effects of AP-1 complex on 5-FU sensitivity based on these results alone. Further studies are warranted to confirm our results.

\section{Acknowledgements}

Not applicable.

\section{Funding}

This work was supported by National Research Foundation of Korea (NRF) grants funded by the Korean government (MSIP) (grant nos. 2017M3C9A5029978, 2018M3A9D3079290, and 2020R1A2C2013258 to HPK).

\section{Availability of data and materials}

The datasets generated and/or analyzed during the current study are available in the Gene Expression Omnibus repository, under the accession number GSE158021.

\section{Authors' contributions}

HPK conceived and coordinated the study. HPK and CMY made substantial contributions to the design and interpretation of data. CMY, MKK, JSJ and YC performed experiments including ATAC-seq, RNA-seq and ChIP-seq. CMY, WJJ and YJK performed bioinformatics analysis. HPK, WJJ and CMY confirmed the authenticity of raw data. HPK and CMY wrote the manuscript. All authors read and approved the final manuscript.

\section{Ethics approval and consent to participate}

Not applicable. 


\section{Patient consent for publication}

Not applicable.

\section{Competing interests}

The authors declare that they have no competing interests.

\section{References}

1. Diasio RB and Harris BE: Clinical pharmacology of 5-fluorouracil. Clin Pharmacokinet 16: 215-237, 1989.

2. Jordan VC: A Retrospective: On clinical studies with 5-fluorouracil. Cancer Res 76: 767-768, 2016.

3. Zhang N, Yin Y, Xu SJ and Chen WS: 5-Fluorouracil: Mechanisms of resistance and reversal strategies. Molecules 13: 1551-1569, 2008.

4. Russo A, Bazan V, Iacopetta B, Kerr D, Soussi T and Gebbia N TP53-CRC Collaborative Study Group: The TP53 colorectal cancer international collaborative study on the prognostic and predictive significance of p53 mutation: Influence of tumor site, type of mutation, and adjuvant treatment. J Clin Oncol 23 $7518-7528,2005$

5. Giovannetti E, Backus HH, Wouters D, Ferreira CG, van Houten VM, Brakenhoff RH, Poupon MF, Azzarello A, Pinedo HM and Peters GJ: Changes in the status of p53 affect drug sensitivity to thymidylate synthase (TS) inhibitors by altering TS levels. Br J Cancer 96: 769-775, 2007.

6. Dominijanni A and Gmeiner WH: Improved potency of F10 relative to 5-fluorouracil in colorectal cancer cells with p53 mutations. Cancer Drug Resist 1: 48-58, 2018.

7. Moll UM, Marchenko $\mathrm{N}$ and Zhang XK: p53 and Nur77/TR3-transcription factors that directly target mitochondria for cell death induction. Oncogene 25: 4725-4743, 2006.

8. Leu JI, Dumont P, Hafey M, Murphy ME and George DL: Mitochondrial p53 activates Bak and causes disruption of a Bak-Mcl1 complex. Nat Cell Biol 6: 443-450, 2004

9. Mihara M, Erster S, Zaika A, Petrenko O, Chittenden T, Pancoska P and Moll UM: p53 has a direct apoptogenic role at the mitochondria. Mol Cell 11: 577-590, 2003.

10. Dall'Agnese A, Caputo L, Nicoletti C, di Iulio J, Schmitt A, Gatto S, Diao Y, Ye Z, Forcato M, Perera R, et al: Transcription factor-directed re-wiring of chromatin architecture for somatic cell nuclear reprogramming toward trans-differentiation. Mol Cell 76: 453-472.e8, 2019.

11. Paliou C, Guckelberger P, Schöpflin R, Heinrich V, Esposito A, Chiariello AM, Bianco S, Annunziatella C, Helmuth J, Haas S, et al: Preformed chromatin topology assists transcriptional robustness of Shh during limb development. Proc Natl Acad Sci USA 116: 12390-12399, 2019.

12. Sobczak M, Pitt AR, Spickett CM and Robaszkiewicz A: PARP1 Co-regulates EP300-BRG1-dependent transcription of genes involved in breast cancer cell proliferation and DNA repair. Cancers (Basel) 11: 1539, 2019.

13. Zhou ZH, Wang QL, Mao LH, Li XQ, Liu P, Song JW, Liu X, $\mathrm{Xu}$ F, Lei J and He S: Chromatin accessibility changes are associated with enhanced growth and liver metastasis capacity of acid-adapted colorectal cancer cells. Cell Cycle 18: 511-522, 2019.

14. Vymetalkova V, Vodicka P, Vodenkova S, Alonso S and Schneider-Stock R: DNA methylation and chromatin modifiers in colorectal cancer. Mol Aspects Med 69: 73-92, 2019.

15. Uusi-Mäkelä J, Afyounian E, Tabaro F, Häkkinen T, Lussana A, Shcherban A, Annala N, Nurminen R, Kivinummi K, Tammela TLJ, et al: Chromatin accessibility analysis uncovers regulatory element landscape in prostate cancer progression. bioRxiv, 2020.

16. Hankey W, Chen Z and Wang Q: Shaping chromatin states in prostate cancer by pioneer transcription factors. Cancer Res 80 2427-2436, 2020.

17. Braadland PR and Urbanucci A: Chromatin reprogramming as an adaptation mechanism in advanced prostate cancer. Endocr Relat Cancer 26: R211-R235, 2019.

18. Arase M, Tamura Y, Kawasaki N, Isogaya K, Nakaki R, Mizutani A, Tsutsumi S, Aburatani H, Miyazono K and Koinuma D: Dynamics of chromatin accessibility during TGF- $\beta$-induced EMT of Ras-transformed mammary gland epithelial cells. Sci Rep 7: 1166, 2017.
19. Rubbi CP and Milner J: p53 is a chromatin accessibility factor for nucleotide excision repair of DNA damage. EMBO J 22: 975-986, 2003

20. Bunz F, Dutriaux A, Lengauer C, Waldman T, Zhou S, Brown JP, Sedivy JM, Kinzler KW and Vogelstein B: Requirement for p53 and p21 to sustain G2 arrest after DNA damage. Science 282: 1497-1501, 1998.

21. Park JH, Choi Y, Song MJ, Park K, Lee JJ and Kim HP: Dynamic long-range chromatin interaction controls expression of IL-21 in CD4+ T Cells. J Immunol 196: 4378-4389, 2016.

22. Buenrostro JD, Wu B, Chang HY and Greenleaf WJ: ATAC-seq: A method for assaying chromatin accessibility genome-wide. Curr Protoc Mol Biol 109: 21.29.1-21.29.9, 2015.

23. Li H and Durbin R: Fast and accurate long-read alignment with burrows-wheeler transform. Bioinformatics 26: 589-595, 2010.

24. Li H, Handsaker B, Wysoker A, Fennell T, Ruan J, Homer N, Marth G, Abecasis G and Durbin R; 1000 Genome Project Data Processing Subgroup: The sequence alignment/map format and SAMtools. Bioinformatics 25: 2078-2079, 2009.

25. Gaspar JM: Improved peak-calling with MACS2. bioRxiv, 2018.

26. Ramirez F, Ryan DP, Grüning B, Bhardwaj V, Kilpert F, Richter AS, Heyne S, Dündar F and Manke T: deepTools2: A next generation web server for deep-sequencing data analysis. Nucleic Acids Res 44 (W1): W160-W165, 2016.

27. Quinlan AR: BEDTools: The Swiss-Army tool for genome feature analysis. Curr Protoc Bioinformatics 47: 11.12.1-34, 2014.

28. Robinson JT, Thorvaldsdóttir H, Winckler W, Guttman M, Lander ES, Getz G and Mesirov JP: Integrative genomics viewer. Nat Biotechnol 29: 24-26, 2011

29. Dobin A, Davis CA, Schlesinger F, Drenkow J, Zaleski C, Jha S, Batut P, Chaisson $M$ and Gingeras TR: STAR: Ultrafast universal RNA-seq aligner. Bioinformatics 29: 15-21, 2013.

30. Li B and Dewey CN: RSEM: Accurate transcript quantification from RNA-Seq data with or without a reference genome. BMC Bioinformatics 12: 323, 2011.

31. Love MI, Huber W and Anders S: Moderated estimation of fold change and dispersion for RNA-seq data with DESeq2. Genome Biol 15: 550, 2014

32. Chen EY, Tan CM, Kou Y, Duan Q, Wang Z, Meirelles GV, Clark NR and Ma'ayan A: Enrichr: Interactive and collaborative HTML5 gene list enrichment analysis tool. BMC Bioinformatics 14: 128, 2013.

33. Kuleshov MV, Jones MR, Rouillard AD, Fernandez NF, Duan Q, Wang Z, Koplev S, Jenkins SL, Jagodnik KM, Lachmann A, et al: Enrichr: A comprehensive gene set enrichment analysis web server 2016 update. Nucleic Acids Res 44 (W1): W90-W97, 2016.

34. Hientz K, Mohr A, Bhakta-Guha D and Efferth T: The role of p53 in cancer drug resistance and targeted chemotherapy. Oncotarget 8: 8921-8946, 2017.

35. Munawar U, Roth M, Barrio S, Wajant H, Siegmund D, Bargou RC, Kortüm KM and Stühmer T: Assessment of TP53 lesions for p53 system functionality and drug resistance in multiple myeloma using an isogenic cell line model. Sci Rep 9: $18062,2019$.

36. Miyamoto K, Nguyen KT, Allen GE, Jullien J, Kumar D, Otani T, Bradshaw CR, Livesey FJ, Kellis M and Gurdon JB: Chromatin accessibility impacts transcriptional reprogramming in oocytes. Cell Rep 24: 304-311, 2018.

37. Penalosa-Ruiz G, Bright AR, Mulder KW and Veenstra GJC: The interplay of chromatin and transcription factors during cell fate transitions in development and reprogramming. Biochim Biophys Acta Gene Regul Mech 1862: 194407, 2019.

38. Matilainen O, Sleiman MSB, Quiros PM, Garcia SMDA and Auwerx J: The chromatin remodeling factor ISW-1 integrates organismal responses against nuclear and mitochondrial stress. Nat Commun 8: 1818, 2017.

39. Weaver IC, Korgan AC, Lee K, Wheeler RV, Hundert AS and Goguen D: Stress and the emerging roles of chromatin remodeling in signal integration and stable transmission of reversible phenotypes. Front Behav Neurosci 11: 41, 2017.

40. Fischer M: Census and evaluation of p53 target genes. Oncogene 36: 3943-3956, 2017

41. Andrysik Z, Galbraith MD, Guarnieri AL, Zaccara S, Sullivan KD, Pandey A, MacBeth M, Inga A and Espinosa JM: Identification of a core TP53 transcriptional program with highly distributed tumor suppressive activity. Genome Res 27: 1645-1657, 2017.

42. Younger ST and Rinn JL: p53 regulates enhancer accessibility and activity in response to DNA damage. Nucleic Acids Res 45: 9889-9900, 2017. 
43. Angel P and Karin M: The role of Jun, Fos and the AP-1 complex in cell-proliferation and transformation. Biochim Biophys Acta 1072: 129-157, 1991.

44. Eferl R and Wagner EF: AP-1: A double-edged sword in tumorigenesis. Nat Rev Cancer 3: 859-868, 2003.

45. Podar K, Raab MS, Tonon G, Sattler M, Barilà D, Zhang J, Tai YT, Yasui H, Raje N, DePinho RA, et al: Up-regulation of c-Jun inhibits proliferation and induces apoptosis via caspase-triggered c-Abl cleavage in human multiple myeloma. Cancer Res 67: 1680-1688, 2007.

46. Bossy-Wetzel E, Bakiri L and Yaniv M: Induction of apoptosis by the transcription factor c-Jun. EMBO J 16: 1695-1709, 1997.

47. Stik G, Vidal E, Barrero M, Cuartero S, Vila-Casadesús M, Mendieta-Esteban J, Tian TV, Choi J, Berenguer C, Abad A, et al: CTCF is dispensable for immune cell transdifferentiation but facilitates an acute inflammatory response. Nat Genet 52: 655-661, 2020.

48. Parikh N, Hilsenbeck S, Creighton CJ, Dayaram T, Shuck R, Shinbrot E, Xi L, Gibbs RA, Wheeler DA and Donehower LA: Effects of TP53 mutational status on gene expression patterns across 10 human cancer types. J Pathol 232: 522-533, 2014.
49. Herrero AB, Rojas EA, Misiewicz-Krzeminska I, Krzeminski P and Gutierrez NC: Molecular mechanisms of p53 deregulation in cancer: An overview in multiple myeloma. Int J Mol Sci 17: 2003, 2016

50. Shaulian E and Karin M: AP-1 in cell proliferation and survival. Oncogene 20: 2390-2400, 2001.

51. Obier N, Cauchy P, Assi SA, Gilmour J, Lie-A-Ling M, Lichtinger M, Hoogenkamp M, Noailles L, Cockerill PN, Lacaud G, et al: Cooperative binding of AP-1 and TEAD4 modulates the balance between vascular smooth muscle and hemogenic cell fate. Development 143: 4324-4340, 2016.

52. Gazon H, Barbeau B, Mesnard JM and Peloponese JM Jr: Hijacking of the AP-1 signaling pathway during development of ATL. Front Microbiol 8: 2686, 2018.

(c) (7) $(9)$ This work is licensed under a Creative Commons C. Atribution-NonCommercial-NoDerivatives 4.0 International (CC BY-NC-ND 4.0) License. 